\section{Depressão entre puérperas: prevalência e fatores associados}

\author{
Postpartum depression: prevalence and \\ associated factors
}

\section{Depresión entre puérperas: prevalencia y factores asociados}

\section{Resumo}

Buscou-se identificar a prevalência e os fatores associados à ocorrência de depressão entre puérperas residentes em um município de médio porte no extremo Sul do Brasil, durante todo o ano de 2013. Entrevistadoras treinadas aplicaram questionário padronizado em todas as participantes nas duas únicas maternidades do município. Investigaram-se características demográficas, socioeconômicas, comportamentais, de suporte social e morbidades. $O$ rastreamento da depressão foi realizado em até 48 horas do puerpério imediato, mediante a utilização da Escala de Edimburgo, sendo o ponto de corte $\geq 10 . \mathrm{Na}$ análise multivariada, utilizou-se a regressão de Poisson com variância robusta. Das 2.687 mulheres entrevistadas, 14\% (IC95\%: 12,9-15,6) foram identificadas com depressão. Fatores como depressão anterior, tristeza no último trimestre da gravidez e historia de depressão na família estiveram associados à maior risco para depressão, assim como ter menor idade e ser multipara. $O$ suporte social fornecido à gestante pela equipe de saúde foi um importante fator de proteção, reduzindo em até $23 \%$ a razão de prevalência de a puérpera desenvolver depressão. Esses resultados indicam a necessidade de incrementar ações por parte dos serviços de saúde em atenção à gestante, a fim de proverlhe maior cuidado nesse momento tão delicado.

Depressão; Período Pós-parto; Diagnóstico; Apoio Social
Juliana Mano Hartmann 1 Raul Andrés Mendoza-Sassi 1 Juraci Almeida Cesar 1

doi: 10.1590/0102-311X00094016
Correspondência

J. M. Hartmann

Universidade Federal do Rio Grande.

Rua General Osório s/n, sala 411, Rio Grande, RS 96201-900, Brasil.

ju_hart@yahoo.com.br

1 Universidade Federal do Rio Grande, Rio Grande, Brasil. 


\section{Introdução}

De acordo com a Organização Mundial da Saúde (OMS), no ano de 2004 a depressão foi considerada a terceira causa de morbidade no mundo, podendo ser a primeira em 2030 1. A literatura indica que as mulheres apresentam um risco duas vezes maior para desenvolver depressão do que os homens, sendo esta diferença ainda mais enfatizada na fase da vida em que se veem responsáveis pelo cuidado de seus filhos 2 . O período gravídico-puerperal é considerado de alto risco para o desenvolvimento de depressão e ansiedade devido às transformações em nível hormonal, físico e emocional vivenciadas pela mulher 2 .

No período gestacional, a depressão apresenta grande variação em sua prevalência entre os países, sendo maior naqueles menos desenvolvidos 3. As taxas de prevalência da depressão durante a gravidez encontradas nos estudos de países em desenvolvimento, incluindo os trabalhos nacionais, estiveram, em sua maioria, por volta de $20 \% 4,5$. Nos países desenvolvidos, a prevalência encontrada variou de $5 \%$ a $30 \% 4$. De acordo com a revisão da literatura, a análise mais detalhada dos estudos realizados no Brasil indica que cerca de 30 a 40\% das mulheres atendidas em unidades básicas de saúde (UBS), na Estratégia Saúde da Família (ESF) ou com perfil socioeconômico baixo, apresentaram alto índice de sintomas depressivos. Por outro lado, pesquisas de base populacional e as que enfocam mulheres assistidas em hospitais identificaram que aproximadamente $20 \%$ delas apresentavam-se deprimidas 6 . Há, também, um estudo indicando que mulheres com história prévia de depressão apresentam risco 50\% maior de desenvolver essa enfermidade no puerpério ${ }^{7}$. Em mulheres com história prévia de depressão pós-parto (DPP), observa-se risco 70\% maior de desenvolverem outro episódio depressivo, e em casos de DPP prévia e melancolia da maternidade, baby blues, este risco aumenta para $85 \% 8$.

Segundo dados da literatura, mães com menor escolaridade, que não residem com o companheiro, não são primigestas, que idealizaram aborto, fizeram uso de álcool/tabaco, sofreram algum evento estressor, tiveram depressão anterior e depressão na família, apresentam maior probabilidade de desenvolver depressão 8,9,10,11,12. Nos estudos sobre o tema, observa-se que a avaliação da depressão pode ser realizada em fases diferentes do ciclo gravídico-puerperal. No entanto, há evidência suficiente de que quanto mais precoce for identificada a patologia, maiores serão as chances de prevenir o agravamento dos sintomas e, por conseguinte, de proteger o desenvolvimento do vínculo mãe/bebê.

Apesar dos diversos trabalhos realizados, existem ainda algumas inconsistências a respeito dos efeitos de determinados fatores de risco, como a idade materna, por exemplo. Outros, ainda não foram suficientemente estudados, como o apoio que a gestante possa receber durante a gestação e o parto vindo por parte dos profissionais de saúde. O suporte social pode ser identificado a partir da integração social do sujeito, por meio da rede de serviços e sensação de que pessoas próximas estão acessíveis, contribuindo para que se sinta seguro e amparado 13. A falta desse apoio durante a gestação, seja da família, do companheiro ou de amigos, aumenta o risco para a ocorrência de depressão 14 . Dentro dessa temática, pouco se fala sobre o suporte fornecido pela equipe de saúde que atende o parto, não estando este relacionado ao aspecto técnico inerente a esta equipe. Em um contexto em que a humanização do parto está sendo discutida e promovida tanto no Brasil 15 como em outros países (OMS), o efeito que o suporte recebido pela gestante, durante o parto por parte da equipe de saúde que a atende, pode ter sobre a depressão, ainda precisa ser mais estudado.

Este trabalho teve por objetivo medir a prevalência e identificar fatores associados à ocorrência de depressão entre puérperas residentes em um município de médio porte no extremo Sul do país.

\section{Metodologia}

Os dados aqui apresentados fazem parte de um estudo mais amplo denominado Estudo Perinatal, que tem por objetivo avaliar a cada três anos a assistência à gestação e ao parto no Município de Rio Grande, Rio Grande do Sul, Brasil. A primeira avaliação foi realizada em 2007, posteriormente, em 2010 e 2013, de onde foram retirados os dados aqui apresentados.

Foram incluídas neste estudo todas as parturientes atendidas nas duas únicas maternidades do município (Santa Casa de Misericórdia de Rio Grande e Hospital Universitário da Universidade 
Federal do Rio Grande - FURG) entre 1o de janeiro e 31 de dezembro de 2013. As crianças nascidas no domicílio também foram incluídas, sendo todos os nascimentos ocorridos em área urbana ou rural rastreados pelo Sistema de Informações sobre Nascidos Vivos (SINASC). Foram excluídas as que não residiam no município e aquelas cujos bebês nasceram com peso inferior a $500 \mathrm{~g}$ ou idade gestacional inferior a 20 semanas.

Este trabalho teve delineamento transversal, censitário. Para o cálculo do tamanho da amostra, realizado a posteriori, utilizou-se nível de $95 \%$ de confiança, poder estatístico de $80 \%$, risco relativo de 2,0, razão de não expostos/expostos de 9:1 (sendo o fator de risco a história anterior de depressão) e prevalência estimada do desfecho nos não expostos de $5 \%$. Com esses parâmetros, o tamanho da amostra necessária, acrescida de $10 \%$ para perdas e $15 \%$ para fatores de confusão, seria de 2.689 gestantes, número muito semelhante ao disponível (2.687).

A coleta das informações ocorreu em até 48 horas após o parto, por meio da aplicação de questionário único, pré-codificado. As variáveis investigadas foram: (a) demográficas: idade, cor da pele referida pela mãe, número de moradores no domicílio; (b) socioeconômicas: escolaridade materna e renda familiar, posteriormente trabalhada como renda per capita da família e em quartis; (c) psicossociais: depressão na família, depressão anterior (“A Sra. já teve depressão alguma vez na vida?"), tristeza no último trimestre de gravidez ("Nos últimos três meses da gravidez a senhora se sentiu triste ou deprimida?", nunca/às vezes/a maior parte do tempo/sempre); (d) comportamentais: uso de álcool e tabaco, e uso de drogas ilícitas; (e) obstétricas: paridade, planejamento familiar e pré-natal; (f) suporte social: investigado mediante pergunta dirigida à gestante sobre como sentiu o apoio recebido por parte do pai do recém-nascido, e medido utilizando-se a escala Likert e posteriormente dicotomizado em não/ruim/regular e bom/ótimo; (g) suporte social profissional: como a puérpera avaliou as informações e apoio emocional, fornecidos a ela pela equipe de saúde durante internação e parto, medidas da mesma forma que na categoria anterior; e (i) morbidade e intercorrências na gestação atual: internação e número de doenças apresentadas durante a gestação, categorizadas com base em uma lista de problemas de saúde como sangramento, descolamento prematuro de placenta, diabetes mellitus e hipertensão.

Os sintomas de depressão foram rastreados usando-se a Escala de Depressão Pós-parto de Edimburgo (EPDS), sendo a mais indicada para esta finalidade por ser de fácil e rápida aplicação 16 . Consiste de dez itens compostos por quatro possibilidades de respostas ( 0 a 3 ), de acordo com a presença ou intensidade do sintoma, tendo como referência os sete dias anteriores à entrevista. O desfecho depressão foi medido mediante o uso da escala, considerando-se escore $\geq 10$ conforme estudo de validação realizado em uma amostra brasileira 17 .

Para este trabalho foram selecionadas e treinadas dez entrevistadoras para a coleta dos dados. O treinamento consistiu na leitura do questionário e do manual de instruções. O estudo piloto foi realizado na primeira quinzena de dezembro de 2012. Todos os questionários foram codificados e revisados pelos entrevistadores e duplamente digitados, em ordem inversa e por diferentes digitadores usando-se o Epidata 3.1 (Epidata Assoc., Odense, Dinamarca).

$\mathrm{Na}$ análise descritiva foi realizado o cálculo de média e desvio padrão (DP) das variáveis contínuas e as proporções das variáveis categóricas. Foi calculada a prevalência de DPP e o intervalo de 95\% de confiança (IC95\%). A análise bivariada foi realizada para o cálculo das razões de prevalência brutas (RP) e IC95\% dos diversos fatores e o desfecho. Para a obtenção das medidas de efeito independentes dos fatores estudados, foi realizada análise multivariada a fim de ajustar os fatores de confusão, utilizando-se a regressão de Poisson com ajuste robusto da variância e cálculo das RP ajustadas. O teste estatístico utilizado nessa fase foi o de Wald. No caso de variáveis ordinais com RP ordenadas, foi usado o teste de tendência linear. Não havendo ordenamento, foi aplicado o teste de heterogeneidade. $\mathrm{O}$ valor de $\mathrm{p}$ utilizado para critério de significância foi $\mathrm{p}<0,05$, de um teste bicaudal. Toda a análise estatística foi conduzida usando-se o programa Stata 13.0 (StataCorp LP, College Station, Estados Unidos).

A análise ajustada foi realizada obedecendo a um modelo hierárquico previamente definido com três níveis. No primeiro, mais distal, foram incluídas as variáveis demográficas e socioeconômicas das puérperas; no segundo nível, variáveis relativas aos fatores psicossociais, comportamentais, de suporte social, obstétricas e pré-natais; no terceiro, variáveis referentes à internação e número de morbidades apresentadas durante a gestação. 
Todas as participantes, ou o seu responsável, assinaram Termo de Consentimento Livre e Esclarecido. O projeto foi submetido e aprovado pelo Comitê de Ética em Pesquisa da Faculdade de Medicina da FURG, sob o número de parecer 85/2012.

\section{Resultados}

Segundo dados do SINASC/Sistema Nacional de Atendimento Médico (SINAM), no ano de 2013 houve 2.769 nascimentos cujas mães residiam no Município de Rio Grande. Neste estudo, 2.687 mães foram identificadas e incluídas, sendo a taxa de respondentes de $97 \%$ (ou $3 \%$ de perdas).

A Tabela 1 mostra que a maioria das mulheres tinha idades entre 13 e 24 anos, 66\% eram de cor da pele branca, parda ou mulata; 44,7\% possuíam de 9 a 11 anos de estudos, 47\% eram primíparas e 86\% moravam com o companheiro. A renda familiar per capita média foi de R \$ 849,65 (DP = 1.000,00). Com respeito aos fatores psicossociais e comportamentais, metade das mulheres não planejou a gestação; cerca de 70\% e 90\% consideraram ótimo o apoio oferecido pelo pai da criança e pela equipe de saúde, respectivamente. Quarenta por cento das puérperas entrevistadas referiram história de depressão na família, e 30\% relataram sentimento de tristeza nos três últimos meses da gestação. Quase a totalidade das puérperas realizou pré-natal.

A Tabela 2 mostra a prevalência de depressão por categoria da variável incluída no modelo e a análise bruta e ajustada. Ter mais idade, maior escolaridade e residir com o companheiro foram fatores de proteção para depressão, já a paridade maior ou igual a dois foi fator de risco. Quanto aos fatores psicossociais, manteve-se associado ao desfecho a depressão em algum momento durante a gravidez, nos últimos três meses da gestação e ocorrência de depressão na família. O maior efeito foi observado nas mulheres que referiram tristeza no último trimestre gestacional $(\mathrm{RP}=4,67)$. Em relação aos fatores comportamentais, o planejamento da gravidez foi fator de proteção e reduziu o risco em $27 \%$. Ter usado tabaco durante a gestação aumentou em $26 \%$ o risco para depressão. O suporte paterno, após o ajuste, perdeu efeito. No que diz respeito aos fatores de exposição de suporte social profissional, tanto ter tido apoio da equipe quanto ter sido acompanhada durante a internação foram fatores de proteção. Aquelas que consideraram o apoio da equipe no momento do parto como bom ou ótimo apresentaram uma redução do risco de 23\%. Quanto ao acompanhamento ao longo da internação, foi observado um aumento do efeito protetor na medida em que este era intensificado, havendo uma redução de $18 \%$ quando era parcial e de 53\% quando foi permanente, comparando-se às que não foram acompanhadas. Finalmente, os eventos mórbidos (internação e número de morbidades) aumentaram o risco de depressão, conforme se identifica na Tabela 2. Observa-se a presença de tendência linear, sendo que o grupo com uma morbidade aumentou o risco em 55\% e aquele com duas ou mais em $127 \%$.

\section{Discussão}

Uma em cada sete gestantes foi afetada por depressão. Os fatores protetores que se mostraram significativamente associados a essa condição foram suporte profissional e apoio da equipe de saúde durante o parto, enquanto que ser adolescente, ter maior paridade e histórico de depressão prévia ou na família foram considerados fatores de risco.

A prevalência de depressão encontrada (14\%) é semelhante, embora inferior, à identificada em estudo realizado no Município de Pelotas, Rio Grande do Sul (16,5\%) 10. Em outros trabalhos, a prevalência variou de 7,2\% a 39,4\% 7,18,19,20,21. A divergência entre as prevalências encontradas pode ser explicada pela diferença no momento da aferição, no ponto de corte utilizado na EPDS, no instrumento usado para identificar a depressão, além dos fatores culturais e socioeconômicos da região em que a pesquisa foi realizada.

Quanto aos fatores socioeconômicos e demográficos, os achados confirmam os resultados de outros estudos. A idade, a multiparidade, a escolaridade, residir com marido/companheiro foram fatores associados à depressão. Identificamos maior risco entre as gestantes multíparas (dois ou mais partos). Em um estudo conduzido no Município de Vitória (Espírito Santo), a multiparidade também surgiu como fator de risco significativo para o desenvolvimento de depressão ${ }^{19}$, o que pode ser expli- 
Tabela 1

Características e fatores associados à depressão (EPDS $\geq 10$ ) em puérperas residentes no Município de Rio Grande, Rio Grande do Sul, Brasil, $2013(\mathrm{~N}=2.687)$.

\begin{tabular}{|c|c|c|c|c|}
\hline Variável & $\begin{array}{c}\text { Descrição da amostra } \\
\text { n (\%) }\end{array}$ & $\begin{array}{c}\text { EPDS }<10 \\
\text { Prevalência (\%) }\end{array}$ & $\begin{array}{c}\text { EPDS } \geq 10 \\
\text { Prevalência (\%) }\end{array}$ & Valor de $p$ \\
\hline Idade materna (em anos) [ $\mathrm{n}=2.685]$ & & & & 0,176 \\
\hline $13-24$ & $1.171(43,6)$ & $995(85,0)$ & $176(15,0)$ & \\
\hline $25-29$ & $648(24,1)$ & $570(88,0)$ & $78(12,0)$ & \\
\hline 30 ou mais & $866(32,3)$ & $737(85,0)$ & $129(15,0)$ & \\
\hline Cor da pele (referida) & & & & 0,005 \\
\hline Branca/Outras & $1.776(66,2)$ & $1.547(87,0)$ & $229(13,0)$ & \\
\hline Preta & $909(33,8)$ & $755(83,0)$ & $154(17,0)$ & \\
\hline Número de filhos & & & & 0,001 \\
\hline Nenhum (primípara) & $1.262(47,0)$ & $1.145(90,7)$ & $117(9,3)$ & \\
\hline 1 & $834(31,1)$ & $716(85,9)$ & $118(14,1)$ & \\
\hline 2 ou mais & $589(21,9)$ & $441(74,9)$ & $148(25,1)$ & \\
\hline Número de moradores no domicílio & & & & 0,001 \\
\hline 2 & $929(34,6)$ & $853(91,8)$ & $76(8,2)$ & \\
\hline 3 & $807(30,0)$ & $700(86,7)$ & $107(13,3)$ & \\
\hline 4 & $453(16,9)$ & $364(80,3)$ & $89(19,6)$ & \\
\hline 5 ou mais & $498(18,5)$ & $387(77,7)$ & $111(22,3)$ & \\
\hline Escolaridade materna (em anos) & & & & 0.001 \\
\hline $1-8$ & $1.064(39,6)$ & $856(80,5)$ & $208(19,5)$ & \\
\hline $9-11$ & $1.201(44,7)$ & $1.061(88,3)$ & $140(11,7)$ & \\
\hline 12 ou mais & $420(15,6)$ & $385(91,7)$ & $35(8,3)$ & \\
\hline Renda familiar (salários mínimos) & & & & 0,001 \\
\hline Até 0.99 & $144(5,4)$ & $558(82,1)$ & $122(17,9)$ & \\
\hline $1,00-1,99$ & $765(28,5)$ & $579(83,5)$ & $114(16,5)$ & \\
\hline $2,00-3,99$ & $1.060(39,5)$ & $555(86,6)$ & $86(13,4)$ & \\
\hline 4,00 ou mais & $716(26,7)$ & $610(90,9)$ & $61(9,1)$ & \\
\hline Reside com marido/companheiro & $2.303(85,8)$ & $1.993(86,5)$ & $310(13,5)$ & 0,003 \\
\hline Já teve depressão ao longo da vida & $446(16,6)$ & $309(69,3)$ & $137(30,7)$ & 0,001 \\
\hline Deprimiu-se nos últimos três meses & $838(31,2)$ & $558(66,6)$ & $280(33,4)$ & 0,001 \\
\hline Depressão na família [n = 2.538] & $1.008(39,7)$ & $811(80,5)$ & $197(19,5)$ & 0,001 \\
\hline Uso de álcool durante a gravidez [n = 2.685] & $251(9,4)$ & $194(77,3)$ & $57(22,3)$ & 0,001 \\
\hline Consumo de tabaco durante a gravidez & $439(16,4)$ & $332(75,6)$ & $107(24,4)$ & 0,001 \\
\hline Uso de outras drogas durante a gestação [n = 2.685] & $23(0,9)$ & $16(69,6)$ & $7(30,4)$ & 0,03 \\
\hline Gravidez planejada & $1.331(49,5)$ & $1.202(90,3)$ & $129(9,7)$ & \\
\hline Apoio da equipe $[n=2.685]$ & & & & $<0,001$ \\
\hline Regular/Ruim & $274(10,2)$ & $209(76,3)$ & $65(23,7)$ & \\
\hline Ótimo/Bom & $2.411(89,8)$ & $2.093(86,8)$ & $318(13,2)$ & \\
\hline Apoio do pai do recém-nascido [ $n=2.562]$ & & & & $<0,001$ \\
\hline Ruim/Regular & $170(6,6)$ & $123(72,4)$ & $47(27,6)$ & \\
\hline Bom & $531(20,7)$ & $441(83,1)$ & $90(16,9)$ & \\
\hline Ótimo & $1.861(72,6)$ & $1.636(87,9)$ & $225(12,1)$ & \\
\hline Gestante foi acompanhada pela equipe de saúde [n = 2.672] & & & & $<0,001$ \\
\hline Não & $61(2,3)$ & $46(75,4)$ & $15(24,6)$ & \\
\hline Parte do tempo & $1.081(40,5)$ & $876(81,0)$ & $205(19,0)$ & \\
\hline Sempre & $1.530(57,3)$ & $1.368(89,4)$ & $162(10,6)$ & \\
\hline Realizou pré-natal & $2.616(97,4)$ & $2.249(86,0)$ & $367(14,0)$ & 0,04 \\
\hline Morbidade na gestação & & & & $<0,001$ \\
\hline 0 & $1.816(67,6)$ & $1.658(91,3)$ & $158(8,7)$ & \\
\hline 1 & $615(22,9)$ & $485(78,9)$ & $130(21,1)$ & \\
\hline 2 ou mais & $256(9,5)$ & $161(62,9)$ & $95(37,1)$ & \\
\hline Hospitalização durante a gestação [n = 2.686] & $240(8,9)$ & $187(77,9)$ & $53(22,1)$ & $<0,001$ \\
\hline
\end{tabular}

EPDS: Escala de Depressão Pós-parto de Edimburgo. 


\section{Tabela 2}

Análises bruta e ajustada para fatores associados à depressão entre puérperas residentes no Município de Rio Grande, Rio Grande do Sul, Brasil, 2013.

\begin{tabular}{|c|c|c|c|c|}
\hline Nível/Variável & RP bruta (IC95\%) & Valor de $p$ & RP ajustada (IC95\%) & Valor de $p$ \\
\hline \multicolumn{5}{|c|}{ I } \\
\hline Idade materna (em anos) & & 0,8 * & & 0,04 * \\
\hline $13-24$ & 1,00 & & 1,00 & \\
\hline $25-29$ & $0,80(0,62-1,03)$ & & $0,70(0,54-0,91)$ & \\
\hline 30 ou mais & $0,99(0,80-1,22)$ & & $0,77(0,61-0,98)$ & \\
\hline Cor da pele & & 0,004 & & \\
\hline Branca/Outras & & & & 0,3 \\
\hline Preta & $1,24(1,00-1,55)$ & & $1,11(0,92-1,35)$ & \\
\hline Número de filhos & & 0,001 & & \\
\hline Primípara & 1,00 & & 1,00 & \\
\hline 1 & $1,52(1,20-1,94)$ & & $1,64(1,28-2,09)$ & 0,001 \\
\hline 2 ou mais & $2,71(2,17-3,38)$ & & $2,80(2,15-3,65)$ & \\
\hline Número de moradores no domicílio & & 0,001 & & 0,08 \\
\hline $1-2$ & 1,00 & & 1,00 & \\
\hline 3 & $1,62(1,22-2,14)$ & & $1,06(0,70-1,60)$ & \\
\hline 4 & $2,40(1,80-3,19)$ & & $1,20(0,81-1,79)$ & \\
\hline 5 ou mais & $2,72(2,07-3,57)$ & & $1,29(0,88-1,88)$ & \\
\hline Escolaridade materna (em anos) & & 0,001 & & $0,02 * *$ \\
\hline $1-8$ & 1,00 & & 1,00 & \\
\hline $9-11$ & $0,59(0,48-0,72)$ & & $0,76(0,61-0,94)$ & \\
\hline 12 ou mais & $0,42(0,30-0,59)$ & & $0,64(0,44-0,92)$ & \\
\hline Renda familiar (quartil) & & 0,001 & & 0,1 \\
\hline Primeiro & 1,00 & & 1,00 & \\
\hline Segundo & $0,97(0,73-1,16)$ & & $1,05(0,83-1,34)$ & \\
\hline Terceiro & $0,74(0,58-0,96)$ & & $0,94(0,72-1,22)$ & \\
\hline Quarto & $0,51(0,38-0,68)$ & & $0,78(0,57-1,09)$ & \\
\hline \multicolumn{5}{|l|}{ II } \\
\hline Com marido/companheiro & $0,70(0,56-0,89)$ & 0,003 & $0,74(0,59-0,93)$ & 0,008 \\
\hline Depressão ao longo da vida & $4,47(3,72-5,36)$ & 0,001 & $1,48(1,2-1,81)$ & 0,001 \\
\hline Tristeza nos últimos três meses & $3,02(2,75-3,32)$ & 0,001 & $4,67(3,67-5,94)$ & 0,001 \\
\hline Depressão na família & $1,88(1,55-2,28)$ & 0,001 & $1,33(1,10-1,61)$ & 0,003 \\
\hline Uso de álcool durante a gravidez & $1,70(1,32-2,18)$ & 0,001 & $0,88(0,69-1,22)$ & 0,3 \\
\hline Fumo durante a gravidez & $1,98(1,62-2,41)$ & 0,001 & $1,26(1,02-1,57)$ & 0,04 \\
\hline Outras drogas durante a gestação & $2,15(1,15-4,02)$ & 0,01 & $0,66(0,32-1,34)$ & 0,2 \\
\hline Gravidez planejada & $1,93(1,58-2,35)$ & 0,001 & $0,73(0,60-0,88)$ & 0,001 \\
\hline Apoio da equipe & & 0,001 & & 0,02 \\
\hline Ruim/Regular & 1,00 & & 1,00 & \\
\hline Ótimo/Bom & $0,56(0,44-0,70)$ & & $0,77(0,61-0,96)$ & \\
\hline Apoio do pai & & 0,001 & & 0,2 \\
\hline Ruim/Regular & 1,00 & & 1,00 & \\
\hline Bom & $0,61(0,45-0,83)$ & & $0,85(0,62-1,16)$ & \\
\hline Ótimo & $0,44(0,33-0,57)$ & & $0,80(0,58-1,10)$ & \\
\hline Gestante foi acompanhada & & 0,001 & & 0,001 * \\
\hline Não teve acompanhamento & 1,00 & & 1,00 & \\
\hline Parte do tempo & $0,77(0,48-1,21)$ & & $0,90(0,57-1,43)$ & \\
\hline Sempre & $0,43(0,27-0,68)$ & & $0,47(0,29-0,74)$ & \\
\hline Realizou pré-natal & $0,62(0,40-0,96)$ & 0,03 & $1,25(0,72-2,16)$ & 0,4 \\
\hline \multicolumn{5}{|l|}{ III } \\
\hline Morbidade na gestação & & 0,001 & & $0,001 * *$ \\
\hline 1 & $2,42(1,96-3,01)$ & & $1,55(1,24-1,94)$ & \\
\hline 2 ou mais & $4,27(3,43-5,31)$ & & $2,27(1,77-2,87)$ & \\
\hline Hospitalização durante a gestação & $1,64(1,26-2,11)$ & 0,001 & $1,27(0,98-1,65)$ & 0,07 \\
\hline
\end{tabular}

* Teste de heterogeneidade;

** Teste de tendência linear. 
cado pelo estresse e sobrecarga na família quando a mulher já tem outros filhos. A maior idade foi fator de proteção, sendo que o menor risco ocorreu em mulheres de 25 anos ou mais. Esse achado é similar ao encontrado em outros estudos realizados 11,22 . Contudo, esse resultado não se repete em outros trabalhos, sendo que alguns não encontraram associação $18 \mathrm{e} e m$ outros a associação foi inversa 23. Dentre os demais fatores socioeconômicos estudados, ressalta-se a escolaridade como fator de proteção, sendo que quanto maior o número de anos completos de estudos, maior é a proteção para depressão, como se observa em outros estudos 11,20. Já em relação à renda, apesar de não ter sido fator associado ao desfecho neste estudo após o ajuste com a escolaridade, em outros se identifica relação entre depressão e baixa renda, sendo esta fator de risco 10,23. Essa maior prevalência de depressão entre os grupos sociais menos privilegiados deixa em evidência a importante iniquidade existente, $\mathrm{e}$ mostra a necessidade de implantar medidas que possam reduzir esta diferença.

Com relação aos fatores psicossociais e comportamentais, a ocorrência de depressão na família e durante a gestação esteve associada à depressão. Identificamos risco três vezes maior entre mulheres que se sentiram tristes ou deprimidas no último trimestre gestacional, e 33\% nas gestantes com história familiar de depressão, sendo ajustados entre si. Tanto ter história de depressão na família 24 quanto indício de depressão na gravidez em curso 21,25 , podem ser fatores que desencadeiam a depressão. Esses resultados indicam que esses fatores, ainda que relacionados, mantêm um efeito independente devendo ser considerados no seu conjunto.

O planejamento da gestação foi fator de proteção para depressão, diminuindo cerca de $30 \%$ o risco de a puérpera desenvolver a doença. Em estudo desenvolvido na cidade de Pelotas, a não aceitação da gravidez foi destacada como um dos fatores que mais influencia o aparecimento de depressão no puerpério. Portanto, planejar a gestação pode possibilitar à mãe maior aceitação da gravidez, colaborando para a diminuição dos riscos de desenvolver depressão 11 . Identificamos uma associação entre o uso de tabaco durante a gravidez e depressão, sendo que o uso de tabaco aumentou em $26 \%$ o risco. Essa relação já foi observada em outras pesquisas sobre o tema 10.

Identificou-se uma associação significativa, com um efeito protetor, entre o suporte oferecido à gestante pela equipe de saúde e a depressão, tanto no que diz respeito à percepção do apoio oferecido pela equipe quanto ao acompanhamento recebido. Esses achados mostram a importância de a gestante ser acolhida durante toda a sua internação, não apenas pela família e amigos, mas também pela equipe de saúde, pois poderia reduzir o risco de depressão 14. Isso parece fortalecer a política de parto humanizado 15 , em que as ações preconizadas e executadas buscam reduzir a possibilidade de manifestação da depressão.

Quanto às intercorrências durante a gestação, verificou-se um aumento progressivo do risco na medida em que aumentava o número de morbidades. Estudos sobre o tema identificaram que mulheres que apresentaram alguma complicação clínica durante a gravidez tiveram maior probabilidade de desenvolver depressão, o que pode ser explicado pela maior fragilidade em que a mulher se encontra ao enfrentar problemas de saúde durante a gestação 20,26 . Vale ressaltar que o efeito dos fatores de suporte profissional e a tendência linear se mantiveram ainda após o ajuste com as variáveis que mediam a morbidade das gestantes. Portanto, esse efeito não ocorreria devido a um maior suporte da equipe pelo fato das pacientes apresentarem necessidade de maiores cuidados.

Ao interpretar esses dados é necessário considerar que o tipo de delineamento deste estudo não permite diferenciar se a depressão surgiu no momento próximo ao parto, configurando o baby blues, se era anterior à gestação ou, ainda, se vai se prolongar no puerpério. Pelo mesmo motivo não é possível descartar a possibilidade de causalidade reversa. Outro aspecto a considerar é que não é possível afastar o viés de recordatório em virtude de tratar-se de um tema delicado, e também da vulnerabilidade da mulher no momento em que a pesquisa foi realizada. A fim de minimizar essa limitação, os questionários eram respondidos de acordo com a disponibilidade da puérpera, respeitando-se o seu momento, sendo a sua participação voluntária. Cabe destacar que o número de perdas foi baixo. Contudo, no caso de algumas variáveis independentes que exigiam o recordatório não imediato, como por exemplo a história de depressão anterior, deve-se considerar a possibilidade da ocorrência de viés de informação, sendo que mulheres com depressão poderiam enfatizar mais sua condição anterior, superestimando o efeito. Ressaltamos, também, que a percepção do suporte social oferecido pelos profissionais pode ser afetada de acordo com a ocorrência de depressão, influenciando a avaliação da gestante sobre o apoio que está recebendo. Ou seja, gestantes deprimidas podem subestimar o apoio que estão recebendo. Um 
último aspecto a considerar é que sendo utilizado um instrumento para rastreamento de depressão, devemos considerar os fatores associados como de risco para um indivíduo identificado com sintomas depressivos, segundo EPDS $\geq 10$, e não necessariamente portador de doença clínica. Essa limitação é própria de todos os estudos que utilizam instrumentos de triagem.

Os dados aqui apresentados mostram a necessidade de novas ações na atenção às gestantes, principalmente durante a realização do pré-natal, visando a oferecer à mulher um acompanhamento mais global de sua gestação, assim como o suporte social e profissional de que necessita naquele momento. A depressão anterior à gestação e a depressão familiar foram fatores de risco para esta doença. Esses fatores podem ser identificados com antecedência e, a partir de um acompanhamento precoce, reduzir o risco dessa condição. Nessa linha de raciocínio, a incorporação de um profissional na área de saúde mental nas equipes de saúde que atendem o pré-natal, assim como nas que atendem o parto no hospital, poderia ser de muita ajuda para o manejo dos casos identificados pelo rastreamento sistemático de depressão durante a gestação e pós-natal. Além disso, deve-se considerar a necessidade de implantar ações voltadas para o acompanhamento da gestante no momento do parto, visando a treinar as equipes de saúde a fim de capacitá-las para oferecerem à mulher o suporte de que necessita neste momento tão delicado, sabendo que este apoio pode ser benéfico também para a redução do risco de depressão puerperal. Da mesma forma, uma vez que essa ocorre após a alta hospitalar da gestante, seria recomendável que o cuidado da equipe profissional se estendesse ao domicílio. Com uma rede de atenção básica bem estruturada como existe no Brasil, esse cuidado poderia ser realizado pelas equipes das UBSs, e em particular pela ESF. Por fim, se os aspectos emocionais da gestante forem considerados pelos profissionais de saúde antes, durante e após o parto, pode haver diminuição da prevalência de depressão, melhorando a qualidade dos vínculos entre a mãe e seu bebê.

\section{Colaboradores}

J. M. Hartmann participou da revisão da literatura, elaboração do projeto, análise e interpretação dos dados e redação do artigo. R. A. Mendoza-Sassi colaborou na elaboração do projeto, análise e interpretação dos dados e redação da versão final do artigo. J. A. Cesar coordenou o projeto que embasou o desenvolvimento do presente trabalho e revisou o artigo.

\section{Agradecimentos}

Agradecemos o apoio oferecido pela Coordenação de Aperfeiçoamento de Pessoal de Nível Superior (Capes), Pastoral da Criança e Secretaria Municipal de Saúde de Rio Grande. 


\section{Referências}

1. World Health Organization. The global burden of disease. 2004 update. http://www.who. int/healthinfo/global_burden_disease/GBD_ report_2004update full.pdf (acessado em 17/ Jun/2015).

2. World Health Organization; United Nations Population Fund. Mental health aspects of women's reproductive health. A global review of the literature. Geneva: WHO Press; 2009.

3. Brito CNO, Alves SV, Ludernir AB, Araujo TVB. Depressão pós-parto entre mulheres com gravidez não pretendida. Rev Saúde Pública 2015; 49:33.

4. Pereira PK, Lovisi GM. Prevalência da depressão gestacional e fatores associados. Rev Psiquiatr Clín (São Paulo) 2008; 35:144-53.

5. Faisal-Cury A, Rossi Menezes P. Prevalence of anxiety and depression during pregnancy in a private setting sample. Arch Womens Ment Health 2007; 10:25-32.

6. Lobato G, Moraes CL, Reichenheim ME. Magnitude da depressão pós-parto no Brasil: uma revisão sistemática. Rev Bras Saúde Matern Infant 2011; 11:369-79.

7. Faisal-Cury A, Menezes PR. Antenatal depression strongly predicts postnatal depression in primary health care. Rev Bras Psiquiatr 2012; 34:446-50.

8. Felix GMA, Gomes APR, França PS. Depressão no ciclo gravídico-puerperal. Comun Ciênc Saúde 2008; 19:51-60.

9. Dois A, Uribe C, Villarroel L, Contreras A. Factores de riesgo asociados a síntomas depresivos post parto en mujeres de bajo riesgo obstétrico atendidas en el sistema público. Rev Med Chile 2012; 140:719-25.

10. Konradt CE. Depressão pós-parto e percepção de suporte social durante a gestação. Rev Psiquiatr Rio Gd Sul 2011; 33:76-9.

11. Silva R, Jansen K, Souza LDM, Moraes IGS, Tomasi E, Silva G, et al. Depression during pregnancy in the Brazilian public health care system. Rev Bras Psiquiatr 2010; 32:139-44.

12. Moraes IGF, Pinheiro RT, Silva RA, Horta BL, Sousa PLR, Faria AD. Prevalência da depressão pós-parto e fatores associados. Rev Saúde Pública 2006; 40:65-70.

13. Baptista MN, Baptista ASD, Torres ECR. Associação entre suporte social, depressão e ansiedade em gestantes. Psic Rev Psicol Vetor Ed 2006; 7:39-48.

14. Corrigan CP, Kawasky AN, Groh CJ. Social support, postpartum depression, and professional assistance: a survey of mothers in the midwestern United States. J Perinat Educ 2015; 24:48-60

15. Secretaria de Políticas de Saúde, Ministério da Saúde. Programa de humanização no pré-natal e nascimento. http://bvsms.saude.gov.br/bvs/ publicacoes/parto.pdf (acessado em 15/Nov/ 2015).
16. Cox JL, Holden JM, Sagovsky R. Development of the 10-item Edimburgh Postnatal Depression Scale. Br J Psychiatry 1987; 150:782-6.

17. Santos IS, Matijasevich A, Tavares BF, Barros AJD, Botelho IP, Lapolli C, et al. Validation of the Edinburgh Postnatal Depression Scale (EPDS) in a sample of mothers from the 2004 Pelotas Birth Cohort Study. Cad Saúde Pública 2007; 23:2577-88.

18. Figueira P, Corrêa H, Malloy-Diniz L, Romano-Silva MA. Escala de depressão pós-natal de Edimburgo para triagem no sistema único de saúde. Rev Saúde Pública 2009; 43 Suppl 1:79-84.

19. Ruschi GEC, Mattar SYSR, Chambô Filho A, Zandonade AE, Lima VJL. Aspectos epidemiológicos da depressão pós-parto em amostra brasileira. Rev Psiquiatr Rio Gd Sul 2007; 29:274-80.

20. Cantilino A, Zambaldi CF, Albuquerque TL, Paes JA, Montenegro ACP, Sougey EB. Post partum depression in Recife, Brazil: prevalence and association with bio-socio-demographic factors. J Bras Psiquiatr 2010; 59:1-9.

21. Tannous L, Gigante DP, Fuchs SC, Busnello ED. Postnatal depression in southtern Brazil: prevalence and its demographic and socioeconomic determinants. BMC Psychiatry 2008; 8:1.

22. Figueiredo B, Bifulco A, Pacheco A, Costa R, Magarinho R. Teenage pregnancy, attachment style, and depression: a comparison of teenage and adult pregnant women in a Portuguese series. Attach Hum Dev 2006; 8:123-38.

23. Edwards RC, Thullen MJ, Isarowong N, Shiu C, Henson L, Hans SL. Supportive relationships and the trajectory of depressive symptoms among young African American mothers. J Fam Psychol 2012; 26:585-94

24. Hedge S, Latha KS, Sripathy MB, Sharma PSVN, Kamath A, Avinash K. Postpartum depression: prevalence and associated factors among women in India. J Womens Health Issues Care 2012; $1: 1$.

25. Silva R, Jansen K, Souza L, Quevedo L, Barbosa L, Moraes I, et al. Sociodemographic risk factors of perinatal depression: a cohort study in the public health care system. Rev Bras Psiquiatr 2012; 34:143-8.

26. Benute GRR, Nomura RMY, Reis JS, Fraguas R, Souza LCS, Zugaib M. Depression during pregnancy in women with a medical disorder: risk factors and perinatal outcomes. Clin Sci 2010; 65:1127-31. 


\section{Abstract}

The aim was to identify the prevalence of postpartum depression and associated factors in women in a medium-sized city in Southern Brazil during 2013. Trained female interviewers applied a standardized questionnaire to all participants in the city's two maternity hospitals. The study analyzed demographic, socioeconomic, behavioral, social support, and disease characteristics. Screening for depression was done within the first 48 hours postpartum, using the Edinburgh Scale with a cutoff of $\geq 10$. Multivariate analysis used Poisson regression with robust variance. Of the 2,687 women interviewed, $14 \%$ (95\%CI: 12.9-15.6) were identified with depression. Factors such as previous depression, third-trimester sadness, and family history of depression were associated with increased risk of postpartum depression, as were younger maternal age and multiparity. Social support from the health team for the pregnant woman was an important protective factor, reducing the prevalence of postpartum depression by 23\%. The results suggest the need for actions by health services for pregnant women in order to provide enhanced care during this delicate phase.

Depression; Postpartum Period; Diagnosis; Social Support

\section{Resumen}

Se buscó identificar la prevalencia, y factores asociados a la ocurrencia de depresión entre puérperas, residentes en un municipio de medio porte en el extremo Sur de Brasil, durante todo el año de 2013. Entrevistadoras entrenadas aplicaron un cuestionario estandarizado a todas las participantes en las dos únicas maternidades del municipio. Se investigaron las características demográficas, socioeconómicas, comportamentales, de apoyo social y morbilidades. El rastreo de la depresión se realizó hasta 48 horas del puerperio inmediato, mediante la utilización de la Escala de Edimburgo, siendo el punto de corte $\geq 10$. En el análisis multivariado, se utilizó la regresión de Poisson con variancia robusta. Da las 2.687 mujeres entrevistadas, un 14\% (IC95\%: 12,9-15,6) se identificaron con depresión. Factores como depresión anterior, tristeza en el último trimestre del embarazo e historia de depresión en la familia estuvieron asociados a un mayor riesgo para la depresión, así como ser menor edad y ser multípara. El apoyo social proporcionado a la gestante por el equipo de salud fue un importante factor de protección, reduciendo en hasta un 23\% la razón de prevalencia de que la puérpera desarrolle depresión. Esos resultados indican la necesidad de incrementar acciones por parte de los servicios de salud en atención a la gestante, con el fin de proveerle un mayor cuidado en ese momento tan delicado.

Depresión; Periodo Posparto; Diagnóstico; Apoyo Social
Recebido em 31/Mai/2016

Versão final reapresentada em 18/Out/2016

Aprovado em 28/Nov/2016 